\title{
Ernstzunehmender Verdacht einer Kindesmisshandlung Bruch der ärztlichen Schweigepflicht kann gerechtfertigt sein
}

Mit Urteil vom 27.06.2013 (Az.: 20 U 19/12) wies das Kammergericht Berlin die Berufung der Mutter eines Kindes gegen einen Krankenhausträger auf Schadensersatz und Schmerzensgeld zurück. Der Krankenhausträger wurde verklagt, weil Mitarbeiter wegen des Verdachts auf Kindesmisshandlung das Jugendamt und das Landeskriminalamt informiert hatten.

\section{Der Fall}

Die Eltern eines Säuglings brachten das Kind wegen eines Krampfanfalls in die Notaufnahme des von der Beklagten betriebenen Krankenhauses. Dort wurde eine subdurale Blutung beidseits sowie Netzhautablösungen beidseits festgestellt. Die Fontanellewarvorgewölbt.NachBehauptungen des Krankenhausträgers soll auch ein Schädelbruch vorgelegen haben, wobei die späteren Kläger bezweifeln, dass die entsprechende Röntgenaufnahme tatsächlich das Kind zeige. Das Kind wurde operativ versorgt und knapp 3 Wochen später aus dem Krankenhaus entlassen. Seitens der Mitarbeiter der Beklagten wurde während des Aufenthalts des Kindes im Krankenhaus gegenüber den Eltern der Verdacht einer Kindesmisshandlung und einem hierdurch ausgelösten „Schütteltrauma“ geäußert. Die Kindeseltern, die die Verletzungen damit erklärt hatten, dass das Kind sich 3 Tage vor Aufnahme ins Krankenhaus nach der Herausnahme des Sitzverkleinerers zur großen Babyschale („Maxi Cosi“) den Kopf gestoßen habe, als der Vater mit dem Auto eine Kurve gefahren sei, verhielten sich zunächst aus Sicht des Krankenhauses kooperativ. Sie lehnten jedoch 4 Tage nach Einlieferung weitere Gespräche mit dem Sozialdienst der Beklagten u.a. ab. Noch am gleichen Tag teilten Mitarbeiter der Beklagten dem Landeskriminalamt Berlin und dem Jugendamt mit, dass bei dem Kind für ein Schütteltrauma typische Verletzungen vorlägen, deren Herkunft ungeklärt sei. In der Folge wurden die Eltern durch die Polizei vorläufig festgenommen. Die Meldung führte darüber hinaus zu einem Ermittlungsverfahren wegen Kindesmisshandlung und zu Maßnahmen zum Schutz des Kindeswohls, wie u.a. zu einer zeitweiligen Unterbringung des Kindes bei Pflegeeltern. Das strafrechtliche Ermittlungsverfahren wurde nach ca. 3 Monaten mangels hinreichendem Tatverdacht eingestellt, da zwar ein Schütteltrauma vorliege, aber nicht festgestellt werden könne, dass das Ereignis stattgefunden habe mit der Folge, dass sich der Verdacht nicht gegen einen bestimmten Täter richten könne. Die Maßnahmen zum Schutz des Kindeswohls wurden rückgängig gemacht. Knapp 3 Jahre später verklagte die Mutter den Krankenhausträger wegen der Anzeigen an das Landeskriminalamt und das Jugendamt durch Mitarbeiter des beklagten Krankenhausträgers auf Schmerzensgeld (für das Kind in Höhe von EUR 25000,00 und für die Mutter in Höhe von EUR 15000,00) und Schadensersatz. Die Klage wurde u.a. damit begründet, dass es keine hinreichenden Anhaltspunkte für eine Kindesmisshandlung gegeben habe.

\section{Die Entscheidung: Verdacht muss ernstzunehmend sein}

Das Landgericht Berlin wies die Klage in erster Instanz nach Einholung eines medizinischen Sachverständigengutachtens ab. Ein Anspruch der Kläger aufgrund einer Verletzung der ärztlichen Schweigepflicht sei nicht gegeben. Es habe der begründete Verdacht einer Kindesmisshandlung bestanden, sodass die Ärzte im wohlverstandenen Interesse des Kindes gehandelt hätten. Gegen dieses Urteil legten die Kläger Berufung ein. Das Kammergericht Berlin gab dem erstinstanzlichen Gericht Recht und wies die Berufung als unbegründet zurück. Nach Auffassung der Richter waren die Ärzte berechtigt, Polizei und Jugendamt einzuschalten, weil aus ex-ante-Sicht ein ernstzunehmender Verdacht einer dem Kind zugefügten Kindesmisshandlung bzw. zumindest vorsätzlichen Körperverletzung bestand und insoweit - was regelmäßig anzunehmen sei - Wiederholungsgefahr bestand. Bei einem ernstzunehmenden Verdacht einer Kindesmisshandlung ist nach Auffassung des erst- und zweitinstanzlichen Gerichts eine Verletzung der ärztlichen Schweigepflicht durch die Information des Landeskriminalamts und des Jugendamts gerechtfertigt. Zur Rechtfertigung müsse die Misshandlung nicht erwiesen sein, ebenso wenig sei ein hinrei- chender Tatverdacht erforderlich, da die Ausermittlung einer möglichen Straftat nicht ärztliche Aufgabe sei. Das Sachverständigengutachten habe ergeben, dass der bei dem Kind unstreitig vorliegende Symptomkomplex klassisch für das Vorliegen eines Schütteltraumas sei und das Schütteltrauma wiederum das klassische Erscheinungsbild einer Kindesmisshandlung im Säuglingsalter darstelle. Alle anderen nach Auffassung der Kläger im Raum stehenden Möglichkeiten seien praktisch auszuschließen. Entscheidend sei, dass die Beklagte angesichts der für ein Schütteltrauma ,typischen" Verletzungen annehmen durfte, dass möglicherweise ein Fall der Kindesmisshandlung vorliegt. Das hätte die Beklagte aus damaliger Sicht auch dann annehmen dürfen, wenn sich im Nachhinein herausgestellt hätte, dass die Verletzungen tatsächlich durch die Fahrweise des Vaters verursacht worden seien. Denn es sei nicht Aufgabe der Ärzte, einen Verdacht ,auszuermitteln“, d.h. definitiv zu klären, welche Ursache eine Verletzung hat. Ausreichend sei es, dass die betreffenden Verletzungen typischerweise durch Kindesmisshandlungen hervorgerufen werden und somit ein „begründeter Verdacht“" vorhanden sei, was aber nicht ausschließt, dass auch noch andere Geschehensabläufe, denen keine Kindesmisshandlung zugrunde liegt, denkbar sind.

Das Kammergericht Berlin macht in den Urteilsgründen den Unterschied zu einem anderen Urteil, in dem Schadensersatz und Schmerzensgeld bejaht wurden, deutlich. In diesem Fall hatte sich ein Kind Hämatome zugezogen, weil es beim Spielen gegen den Türrahmen der Wohnzimmertür gestürzt war. Die Eltern sahen sich auch dort dem Verdacht der Kindesmisshandlung ausgesetzt. Hier gingen die Ansprüche der Kläger durch, da das dort zu entscheidende Gericht davon ausging, dass die Verletzungen des Kindes hätten lebensnah und zwanglos mit der Unfalldarstellung der Eltern erklärt werden können, da derartige „Spielunfälle“ geradezu typisch für Kinder seien.

Wiederholungsgefahr erforderlich Die Richter stellen auch eindeutig klar, dass der Bruch der ärztlichen Schweige- 
pflicht nur dann gerechtfertigt ist, wenn Wiederholungsgefahr im Raum steht und die Ärzte die Behörden informieren, um künftige Gesundheitsschäden vom Kind abzuwenden. Der Heilauftrag umfasse nicht nur das Erkennen und die Behandlung von Erkrankungen, sondern auch die Vermeidung von künftigen Gesundheitsgefährdungen. Lägen mehrere dem Kind vorsätzlich zugefügte Verletzungen vor bzw. ist ein entsprechender Verdacht gegeben, so liege die Wiederholungsgefahr auf der Hand. Aber auch, wenn „nur“ eine vorsätzliche Körperverletzung im Raum stehe, sei - zumindest bei derart schwerwiegenden Verletzungen, die lebensbedrohlich seien - von einer Wiederholungsgefahr auszugehen.

Diskutiert wird im Urteil auch die Frage, inwieweit die Mitarbeiter des Krankenhauses nur das Jugendamt hätten informieren dürfen, nicht aber die Polizei. Hierzu stellen die Richter fest, dass man zwar diskutieren könne, ob es hier „tunlich“ war, auch die Polizei einzuschalten. Den Mitarbeitern der Beklagten sei kein Vorwurf zu machen. Es sei zumindest „rechtlich vertretbar“ gewesen, auch das Landeskriminalamt einzuschalten.

Keine Verantwortung für etwaige „Überreaktion“ der Strafverfolgungsbehörden

Der Senat setzt sich auch damit auseinander, dass er sich durchaus bewusst ist, dass der Verdacht auf Kindesmisshandlung und das rechtlich nicht zu beanstandende Vorgehen der Mitarbeiter des Krankenhauses das persönliche Leben der Eltern nachhaltig beeinflusst und schwerwiegende Folgen für sie ausgelöst habe. Es stellt jedoch klar, dass Kindeseltern, die dem Verdacht einer Kindesmisshandlung ausgesetzt seien, solche Belastungen hinzunehmen haben, welche Maßnahmen auslösten, die in der Absicht getroffen würden, das vermeintlich gefährdete Kindeswohl, welches Vorrang vor den Befindlichkeiten der Eltern habe, zu schützen. Im Übrigen stellten die Richter fest, dass die Beklagte für ein etwaiges Fehlverhalten oder eine „Überreaktion“ der Strafverfolgungsbehörden oder des Jugendamts nicht verantwortlich wären. Insbesondere wäre die Anzeige nicht so abgefasst gewesen, dass man von einer Kindesmisshandlung ausgehen musste.

\section{Hinweise für sensible} "Konfrontationsgespräche ${ }^{\text {" }}$ Des Weiteren gibt das Kammergericht Hin- weise, wie es nach Auffassung der Richter wünschenswert wäre, „Konfrontationsgespräche“ zwischen den Mitarbeitern des Krankenhauses und den Kindeseltern in solchen Fällen zu führen. So regen sie an, dass von Seiten der entsprechenden Mitarbeiter die Gespräche unter dem Blickwinkel geführt werden sollten, dass die Kindeseltern als nicht schuldig anzusehen seien und auch dementsprechend behandelt werden sollten, solange aufgrund von Ermittlungen nicht das Gegenteil feststehe. Gleichermaßen wünschenswert sei auch eine Sensibilisierung dafür, dass selbst ein sich letztlich nicht bestätigender Verdacht einer Kindesmisshandlung für die verdächtigten Kindeseltern gleichwohl schwerwiegende Folgen in verschiedener Hinsicht haben könne. Das Gericht stellt aber auch sehr deutlich klar, dass es sich hierbei um Fragen handele, die die Art und Weise des Umgangs miteinander beträfen und die einer gerichtlichen Bewertung im Rahmen von Schadensersatzansprüchen nicht zugänglich seien. Die Revision gegen das Urteil wurde nicht zugelassen.

\section{Fazit}

Das Kammergericht Berlin hat in einem äußerst sensiblen Bereich eine sensible Entscheidung getroffen. Dennoch darf dieses Urteil nicht als Legitimation für einen leichtfertigen Bruch der Schweigepflicht herangezogen werden. Das Gericht hat sehr deutlich herausgearbeitet, dass es immer eine Einzelfallentscheidung ist, ob eine Verletzung der Schweigepflicht gerechtfertigt ist oder nicht. Deutlich wird, dass es sich um einen begründeten Verdacht handeln muss und mit einer Wiederholungsgefahr zu rechnen ist. Zwischen den Zeilen wird letztlich auch deutlich, dass das Gericht eine Information an das Jugendamt wohl als ausreichend empfunden hätte. Die Einschaltung der Polizei wurde zwar rechtlich nicht beanstandet, aber durchaus als diskutabel dargestellt. Dies deckt sich mit dem Gesetz zur Kooperation und Information im Kinderschutz (KKG). Dort ist in $\S 4$ die Beratung und Übermittlung von Informationen durch Geheimnisträger bei Kindeswohlgefährdung geregelt. Dort ist vorgesehen, dass, wenn u.a. Ärzten in Ausübung ihrer beruflichen Tätigkeit gewichtige Anhaltspunkte für die Gefährdung des Wohls eines Kindes oder eines Jugendlichen bekannt werden, sie mit dem Kind oder Jugendlichen und den Personensorgeberechtigten die Situation erörtern sollen und, soweit er- forderlich, bei den Personensorgeberechtigten auf die Inanspruchnahme von Hilfe hinwirken sollen, soweit hierdurch der wirksame Schutz des Kindes oder des Jugendlichen nicht in Frage gestellt wird. Zur Einschätzung der Kindeswohlgefährdung haben Ärzte gegenüber dem Träger der öffentlichen Jugendhilfe Anspruch auf Beratung durch eine insoweit erfahrene Fachkraft. Sie sind zu diesem Zwecke befugt, dieser Person die dafür erforderlichen Daten zu übermitteln; vor einer Übermittlung der Daten sind diese zu pseudonymisieren (hierbei wird der Name und andere Identifikationsmerkmale durch ein Kennzeichen (z.B. Nummerncode) ersetzt). Scheidet eine Abwendung der Gefährdung bei den vorbeschriebenen Maßnahmen (Gespräch mit den Eltern, Hinwirken auf Inanspruchnahme von Hilfe) aus oder ist ein solches Vorgehen erfolglos und halten die Ärzte ein Tätigwerden des Jugendamts für erforderlich, um eine Gefährdung des Wohls eines Kindes oder eines Jugendlichen abzuwenden, so sind sie befugt, das Jugendamt zu informieren; hierauf sind die Betroffenen vorab hinzuweisen, es sei denn, dass damit der wirksame Schutz des Kindes oder des Jugendlichen infrage gestellt wird. Zu diesem Zweck sind die Ärzte befugt, dem Jugendamt die erforderlichen Daten mitzuteilen.

Die ärztliche Schweigepflicht ist eine Kernpflicht des ärztlichen Berufs. Eine Verletzung derselben muss folglich aus gewichtigen Gründen gerechtfertigt sein. Ob eine solche Rechtfertigung vorliegt, muss jeweils im Einzelfall beurteilt werden. Wie das Urteil zeigt, stehen neben strafrechtlicher Verfolgung mit entsprechenden weiteren Konsequenzen auch Schadensersatzansprüche im Raum. Das Urteil zeigt jedoch auch auf erfreuliche Weise, dass in „Notfällen“ die Schweigepflicht gebrochen werden darf, um weitere Gefahren abzuwenden. Im Zweifelsfall sollte Rechtsrat eingeholt werden. Dies letztlich auch, um abzuklären, inwieweit möglicherweise als strafrechtlicher "Garant" sogar eine Verpflichtung zur Informationsweitergabe bestehen könnte.

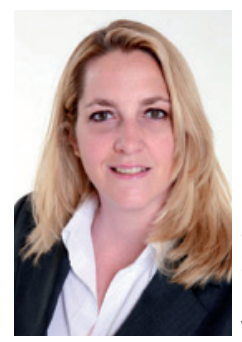

Korrespondenz Dr. iur. Isabel Häser Rechtsanwältin ECOVIS Lüdemann Wildfeuer \& Partner Sonnenstr. 9 80331 München www.ecovis.com 